\title{
CURVATURE AND LENGTH
}

\author{
BY RICHARD JERRARD
}

Communicated by S. Bochner, September 6, 1960

In this note an invariance property of curvature of plane curves is discussed. The property is essentially this: the pointwise curvature of a family $F$ of plane curves integrated over the interior of a simple, closed, smooth curve $B$ depends only upon the angles of intersection of $B$ with members of $F$. The property is exactly stated and proved below, and some of its consequences are discussed. These are first, the connection of the integrated curvature with the boundary length, and second an integral inequality for meromorphic functions.

The results depend upon the following simple lemma.

Lemma A. If $u$ is a real-valued function of class $C^{2}$ defined in the plane, $K(x, y)$ is the curvature at $(x, y)$ of the level curve $u=$ const. through $(x, y)$, and $n$ is the unit normal to $u=$ const. then

$$
\operatorname{div} n=K \text {. }
$$

The proof follows readily by calculation of the divergence of $n$ from

$$
\mathbf{n}=\left(u_{x}, u_{y}\right) /\left(u_{x}^{2}+u_{y}^{2}\right)^{1 / 2},
$$

and comparison with the easily obtained formula.

$$
K=\left(u_{x}^{2} u_{y y}-2 u_{x} u_{y} u_{x y}+u_{y}^{2} u_{x x}\right) /\left(u_{x}^{2}+u_{y}^{2}\right)^{3 / 2} .
$$

We take the sign of $K$ to be defined by this formula.

With this lemma we are able to prove the following theorem on the integrated curvature over a region for such a function.

THEOREM 1. If $G$ is a region in the plane whose boundary $B$ consists of a finite number of simple, closed, piecewise smooth curves, and $u$ is a function of class $C^{2}$ almost everywhere whose level curves have curvature $K(x, y)$, then

$$
\int_{G} K d A=\int_{B} n \cdot N d s,
$$

where $n$ and $N$ are the unit normal vectors to $u=$ const. and $B$.

The proof is an application of Green's Theorem. If, using Lemma A, we replace $K$ by $\operatorname{div} n$ then the above formula is exactly a statement of Green's Theorem. Of course, the conditions placed on the 
region $G$ can be any alternate conditions such that Green's Theorem holds.

This formula shows that the integrated curvature over $G$ is invariant under any change of $u$ which leaves fixed the normal vector $n$ along $B$. We note that the function $u$ may have isolated singularities; the level curves in the neighborhood of a pole are like those in the neighborhood of a simple maximum. Indeed, the theorem holds for any function $v=g(u)$, where $g$ is an arbitrary real-valued function.

As immediate consequences we have the following two corollaries.

Corollary 1. If $B$ is a simple, closed level curve of a class $C^{2}$ function $u$ and $G$ is its interior, then

$$
\int_{G} K d A=L(B)=\text { Length of } B
$$

for then $n \cdot N=1$.

Corollary 2. If $B$ is the boundary of a region $G$ as in Theorem 1 , then

$$
\int_{G} K d A \leqq L(B)
$$

for $n \cdot N \leqq 1$.

We can use the last result to give an application to meromorphic functions.

Theorem 2. If $C$ is the unit circle, then the inequality

$$
\int_{C} \frac{\operatorname{Re}\left(f^{\prime}\right)}{|f|} d A \leqq 2 \pi
$$

holds for any meromorphic function $f$.

Proof. Rubel and the author have recently shown [1] that if $f$ is any meromorphic function, a function $w=u+i v$ defined by $f=1 / w^{\prime}$ has the property that the curvature of the level curves $u=$ const. is given by $K=\operatorname{Re}\left(f^{\prime}\right) /|f|$. The theorem then follows immediately from Corollary 2.

\section{BIBLIOGRAPHY}

1. R. P. Jerrard and L. A. Rubel, A distortion theorem, to appear.

UNIVERSITY OF ILLINOIS 